\title{
Comments
}

\section{Electroanalytical Obscurity: An Alternative View}

\author{
Robert de Levie*
}

Received June 19, 2017; Accepted after Peer-Review July 25, 2017

\begin{abstract}
An alternate view is presented of some of the origins of what Prof. Kakiuchi recently called "obscurity in electroanalytical chemistry".
\end{abstract}

\section{Introduction}

In the same issue of Reviews of Polarography in which Prof. Kenji Kano [1] announced the award of the Heyrovský Medal of the Czech Academy of Sciences to Prof. Takashi Kakiuchi, its recipient commented [2] on my recent review [3] of the historical development of $\mathrm{pH}$, from the groundbreaking publications of Friedenthal [4] and Sørensen [5] to the present. My review had been triggered by comments in the official IUPAC magazine by one of the twelve co-authors of the latest IUPAC-recommended definition of $\mathrm{pH}$ [6], Prof. Camões [7], who wrote that the $\mathrm{pH}$ is

"most likely the most measured chemical parameter"

but that

"beyond the simple process of measuring $\mathrm{pH}$, there is poor understanding of the concept, the basis for its derivation, and limitations of its applicability."

and attempted to explain why that might indeed be the case.

In this context, Prof. Kakiuchi [2] gave his comments the title "Obscurity in Electroanalytical Chemistry". He stated that and that

"Electrochemistry is intrinsically nonthermodynamic, ...",

"The obscurity associated with nonthermodynamic nature of electrochemical cells translates to the obscure interpretation of the Nernst equation of the form $E=E^{0}+(R T / F) \ln \left(a_{\mathrm{Ox}} / a_{\mathrm{Rd}}\right)$, which has long been applied to a working electrode in electroanalytical chemistry. By focusing on this obscurity, it is possible not only to make our understanding of electroanalytical chemistry clearer but to design an electrochemical cell for less obscure, more reliable estimates of single ion activities."

In his conclusions, Prof. Kakiuchi then embraced this obscurity as follows:

\footnotetext{
* Bowdoin College, Brunswick ME 04011, USA

E-mail: rdelevie@bowdoin.edu
} 


\begin{abstract}
"What is described above is the current stage of obscurity in electroanalytical chemistry. Rather than taking this obscurity as gloomy, "real threat"ening" or "troublesome", we had better treated it as the intrinsic feature of electroanalytical chemistry. By doing so, we may gain a quantitative understanding of the obscurity, and further minimize it."
\end{abstract}

and he also mentioned

"the obscurity in the potential of the working electrode".

Below I will take a different view, viz. that a major goal of science in general, and of science education in particular, is to eliminate obscurity rather than to accept it as "the intrinsic feature of electroanalytical chemistry", because such obscurity can readily lead to imprecise thinking and invalid conclusions. As outlined earlier [3], I view such obscurity as largely resulting from the IUPAC recommendation [6], going back to a paper by Bates \& Guggenheim [8] and an associated decision by IUPAC in 1985 [9], to redefine $\mathrm{pH}$, an eminently practical and frequently measured solution property, from Sørensen's original $-\log \left[\mathrm{H}^{+}\right]$to $-\log \left(a_{\mathrm{H}^{+}}\right)$, while at the same time acknowledging that $a_{\mathrm{H}^{+}}$is immeasurable, not just in practice but in principle! This disconnects $\mathrm{pH}$ measurements not only from any possible thermodynamic foundation, but also from any other connection with general chemical theory and practice. Is there no inherent conflict when one defines $\mathrm{pH}$, an important experimental quantity, in terms of an admittedly [6] immeasurable hydrogen activity $a_{\mathrm{H}^{+}}$? According to Lord Kelvin [10]:

“... when you can measure what you are speaking about, and express it in numbers, you know something about it; but when you cannot measure it, when you cannot express it in numbers, your knowledge of it is of a meager and unsatisfactory kind: it may be the beginning of knowledge, but you have scarcely, in your thoughts, advanced to the stage of science, ..."

I fully agree with Prof. Kakiuchi's [2] implication that electrochemistry, as reflected in some of its papers and textbooks, harbors obscurities, but I do not consider such obscurity as "the intrinsic feature of electroanalytical chemistry", but rather as some eminently correctable misconceptions which, as educators, we should try to clarify rather than excuse. In my view, the IUPACrecommended definition of $\mathrm{pH}$ as $-\log \left(a_{\mathrm{H}^{+}}\right)[6]$ is a major (but by no means the only) contributor to this confusion. However, in its blatant disregard of thermodynamics, it is probably the primary cause of the obscurities identified by Prof. Kakiuchi, especially in view of the status of IUPAC as an authoritative international standardizing organization. A second contributor may well be "the obscurity in the potential of the working electrode", which will be the focus of section 3.

This confusion is amplified by the fact that chemical equilibria, $\mathrm{pH}$, acid-base titrations, and the Nernst equation are most often taught to the next generation of scientists in the introductory (high school and/or first-year college) chemistry courses, whereas the meaning and context of activity corrections in these are usually (if at all) explained several semesters later, within the framework of an undergraduate physical chemistry course covering equilibrium thermodynamics, typically taken by a relatively small fraction of those who took introductory chemistry. Many students are thus left with this mysterious, typically unexplained fudge factor $f$ or $\gamma$ called "ionic activity coefficient". What a shame, especially since acid-base titrations with Sørensen's definition of $\mathrm{pH}$ as $-\log \left[\mathrm{H}^{+}\right]$can be such convincing examples of the quantitative agreement between chemical theory and practice! Below I will explain what may have caused the resulting obscurities. In my opinion these are mainly caused by the IUPAC recommended protocol for 
measuring $\mathrm{pH}$, which totally ignored the important studies by Brønsted between 1914 [11] and 1923 [12] on "swamping", i.e., on using solutions in which the ionic strength is dominated by the presence of a chemically non-interfering, "inert" or "supporting" strong electrolyte of constant concentration. If that approach can be used routinely for other successful electroanalytical methods, such as polarography and cyclic voltammetry, then why not for $\mathrm{pH}$ measurements?

Brønsted first observed that the presence of such a swamping electrolyte at a sufficiently high and constant level rendered the activity coefficients of minor solutes constant, and then found the reason why this is so. This led to the very useful concept of "trace" activity coefficients. In the present context I merely note that swamping by a so-called "supporting" or "inert" strong electrolyte is an integral part of polarography, although the reasons why it works are often only partially understood, and are usually merely ascribed to its lowering of the solution resistance, and may therefore (mistakenly) be considered inapplicable to equilibrium (zero-current) measurements.

Swamping allows the direct potentiometric determination of $-\log \left[\mathrm{H}^{+}\right]$, and hence of the hydrogen ion concentration $\left[\mathrm{H}^{+}\right]$. Near the end of his scientific career, Bates et al. [13-15] finally reached a similar conclusion, and proposed its use for certain aqueous solutions of major scientific and general interest, such as for monitoring the $\mathrm{pH}$ of seawater, but by then he had already retired from his position at NBS, and the IUPAC protocol had already been implemented.

Secondly, obscurity is often rooted in disagreements about how best to teach some of the fundamental relations of electrochemistry, such as the Nernst equation. This often comes down to the question: should we explain difficult concepts in a rather oversimplified way, or explain them correctly to avoid problems later? We will also note that such interpretational differences often run deep, and can sometimes be traced far back. I therefore thank Prof. Kakiuchi for joining the discussion, presenting an opposing point of view, and assembling an impressive collection of such individual obscurities. I look forward to his planned "reflections".

My comments will be offered in the following sequence. Section 2 will consider the role of Gibbsian (i.e., equilibrium) thermodynamics in electrochemistry, and more specifically in potentiometry. Some of the examples given by Prof. Kakiuchi [2] will then be analyzed in sections 3 and 4 .

But first some comments regarding the notation and terminology used. Because experimental data on activities and standard potentials are usually listed in terms of the gravimetric or mola $\boldsymbol{\ell}$ concentrations $m$, I will use that nomenclature here, which has the added advantage of being unaffected by temperature because, contrary to volume, weight does not vary with temperature. On the other hand, the volumetric measure is easier to use in volumetric titrations. In dilute aqueous solutions at room temperature, the difference between the volumetric or molar concentration $c$ (in moles per liter of solution, $\mathrm{mol} \mathrm{L}^{-1}, \mathrm{~mol} / \mathrm{L}$, molar, or simply $\mathrm{M}$ ), and the gravimetric concentration $m$ (the "mola ity" in moles of solute per kilogram of solvent, $\mathrm{mol} \mathrm{kg}{ }^{-1}, \mathrm{~mol} / \mathrm{kg}$, or mola $\ell$ ), is slight and often negligible. And at constant swamping concentrations, it is merely an added constant. We will also encounter a case, see eq. (2.1) in section 2, where it is clearly simpler to work with $m$ rather than with $c$. Otherwise, it doesn't affect the arguments offered below, merely their precise, numerical values. To convert from one convention to the other, use

$$
c=m \times d
$$

where $d$ is the density of the solution (in $\mathrm{kg} \mathrm{L}^{-1}$ ), as is readily verified dimensionally since

$$
c \mathrm{~mol} \mathrm{~L}^{-1}=m \mathrm{~mol} \mathrm{~g}^{-1} \times d \mathrm{~kg}^{-1}=m \times d \mathrm{~mol} \mathrm{~L}^{-1}
$$


In order to keep the arguments simple, and avoid getting mixed-up in non-essential factors, we will restrict our discussion to monovalent ions, i.e., with ionic valences $z_{i}$ of either +1 or -1 . And we will use the term "thermodynamics" in the commonly used physical-chemistry sense of "equilibrium thermodynamics", as emphasized by Gibbs and Lewis \& Randall, again see section 2.

\section{Thermodynamics and potentiometry}

We now turn to section 2 of [2], which opens with

"It is well known that the single ion activity is not measurable."

and I fully agree. However, I am not sure what Prof. Kakiuchi means in his next sentence,

"However, the meaning of this immeasurability is not well comprehended."

In my reading, "not measurable" is quite unambiguous: it means that it cannot be quantified, cannot be given an experimentally well-defined numerical value. See also the above quote from Lord Kelvin [10]. But why follow this with an insinuating, doubt-inducing sentence such as

"... we may tend to have impressions, such as, something dubious, about the ion activity"

rather than pointing to its well-known cause, viz. the charge-balance requirement of macroscopic electroneutrality.

In the next paragraph Prof. Kakiuchi wrote:

"There are of course many physical quantities that are non-thermodynamic, but precisely measurable. The velocity, the rate of acceleration, and other time-dependent physical quantities, are of course measured with high precision."

True enough, but also irrelevant. When Gibbs [16] formulated his magnum opus on chemical thermodynamics, in his two long papers "On the Equilibrium of Heterogeneous Substances", the very title of his seminal work, featuring the key word Equilibrium, showed that he specifically limited his treatment to the equilibrium behavior of heterogeneous systems, thereby excluding all time-dependent processes. In fact he based his entire thermodynamics on the general equilibrium conditions. The symbol $t$, as it is defined in the very first paragraph of his first paper and is used throughout his work, is used to denote the absolute temperature rather than time! "Irreversible" thermodynamics, as subsequently developed by Guggenheim, Onsager, Prigogine and others, deals with kinetic processes such as diffusion, but need not be considered here.

In the present context it may be useful to distinguish clearly between electrochemical equilibrium measurements, such as those of $\mathrm{pH}$, interfacial tension and capacitance, and salt activities, all of which are intrinsically time-independent (but of course can be used in timedependent experiments), and electrochemical kinetic experiments, such as polarography, 
cyclic voltammetry, and other non-zero-current techniques, where time is often an essential experimental factor, e.g., through mass transport and/or electrode kinetics. In typical $\mathrm{pH}$ measurements, as discussed below, we deal exclusively with time-independent, equilibrium measurements.

In his letter to Bancroft, Gibbs [17] explained the paucity of his electrochemical results specifically because only few electrochemical systems are at equilibrium. Using the abbreviation E.H.S. for his thermodynamic papers, Gibbs wrote:

"The meagerness of the results obtained in my E.H.S. in the matter of electrolysis has a deeper reason than the difficulty of the evaluation of the potentials.

In the first place, cases of true equilibrium (even for open circuit) are quite exceptional. Thus the single case of unequal concentrations of the electrolyte cannot be one of equilibrium since the process of diffusion cannot be stopped. Cases in which equilibrium does not subsist were formally excluded in my subject, and indeed could not be satisfactorily treated without the introduction of new ideas foreign to those necessary for the treatment of equilibrium."

Gibbs couldn't have been more explicit!

And Nernst, at the end of his first Silliman Memorial Lecture at Yale University on The Experimental and Theoretical Applications of Thermodynamics to Chemistry [18], stated that:

“... the two laws of thermodynamics are insufficient for a general explanation of nature. They take, for example, no account of the course of phenomena in time, - unlike the molecular theory, in which such a limitation has not thus far been shown to exist."

Gibbsian (i.e., equilibrium) thermodynamics provides a powerful, strictly logical framework based on just two key observations by Clausius [19], viz. that the energy in the world is constant, while its entropy tends to a maximum. From just these two general observations, Gibbs managed to derive mathematical relations between many otherwise seemingly quite unrelated equilibrium parameters, such as "reversible" cell potentials, freezing point depressions, boiling point elevations, osmotic pressures, and isopiestic measurements, and Lewis [20] extended this list by defining electrolyte activities and activity coefficients. Moreover, classical thermodynamics represents a purely macroscopic approach, and neither deals with nor interprets details such as the atomic nature of chemistry, not even the existence of electrons and ions.

There are some inherent constraints on what information equilibrium thermodynamics can provide, specifically the requirement that one can only obtain thermodynamic information about independently manipulable, thermodynamic "components" of the system. Such thermodynamic components are invariably electroneutral substances or mixtures, as readily understood in terms of Poisson's equation [21]. Thus, the mean activities $a_{ \pm}$of strong acids, bases, and salts can be determined experimentally, but those of single ionic species cannot. Why is that so?

Activity is a purely thermodynamic concept, introduced by Lewis [20] as the partial derivative of the total Gibbs free energy $G$ in a closed system, and can therefore be measured 
only when it can be defined as such. Unlike ionic concentrations, ionic activities and activity coefficients are strictly thermodynamic constructs, and have no meaning if they cannot be defined thermodynamically. And when they have neither definition nor meaning, they are indeed immeasurable.

Their thermodynamic definition requires that we can uniquely divide the Gibbs free energy $G$ of a mixture into the partial free energies $\mu_{i}=\mu_{i}{ }^{\circ}+R T \ln a_{i}$ of its thermodynamic "components" in the form of a partial derivative with respect to the number of moles $n_{i}$ of component $i$ in that mixture, keeping the temperature $T$, the pressure $P$, and the amounts $n_{j-i}$ of all other components constant, as in

$$
\mu_{i}=\left(\frac{\partial G}{\partial n_{i}}\right)_{P, T, n_{j \neq i}}
$$

where $n_{i}$ is the number of moles of species $i$. We can do that with solvent mixtures of, e.g., water and ethanol, or with solutes such as sugar, $\mathrm{HCl}, \mathrm{Na}_{2} \mathrm{SO}_{4}$ in their solvent, but since we cannot add macroscopic amounts of, say, only $\mathrm{H}^{+}$to an aqueous $\mathrm{HCl}$ solution, or withdraw just chloride ions from it, thermodynamics doesn't allow us to define the corresponding "ional" partial free energies

$$
\widetilde{\mu}_{i}=\widetilde{\mu}_{i}^{\circ}+R T \ln a_{i}+z_{i} F \psi
$$

which is why Guggenheim [22] warned his readers that the electric potential difference $\Delta \psi$ between two points in different media can never be measured, and that

"it is therefore a conception which has no physical significance".

Consequently, hoping to measure the individual components of (2.2) is wishful thinking or, as Guggenheim [23] wrote:

“... we therefore have no knowledge of the value of the electric potential between any pair of phases, nor therefore of the chemical potential, the activity or the activity coefficient of any individual ion."

This matter was discussed at length in section 1.6 of [3], which extended Guggenheim's special, tilde-based notation to all three unknowables in this case: the chemical potential $\tilde{\mu}_{i}^{\circ}$, the activity $\widetilde{a}_{i}$ of ions, and the potential $\widetilde{\psi}$ of a single phase, as in

$$
\tilde{\mu}_{i}=\tilde{\mu}_{i}^{\circ}+R T \ln \tilde{a}_{i}+z_{i} F \tilde{\psi}
$$

as well as

$$
\widetilde{\gamma}_{i}=\widetilde{a}_{i} / m_{i} \text { or } \tilde{f}_{i}=\widetilde{a}_{i} / c_{i}
$$

in order to alert the user to the absence of physical meaning of related, immeasurable quantities. We might wish it were otherwise, but that will not make it so. And because the Gibbs free energy, its chemical potential, as well as activity and activity coefficients, are strictly thermodynamic concepts, there is no useful distinction between "thermodynamically immeasurable" and just "immeasurable". 
We can weigh $10 \mathrm{~g}$ of ethanol, of sugar, of sodium chloride, of sulfuric acid. But we cannot weigh $10 \mathrm{~g}$ of $\mathrm{Na}^{+}$or $\mathrm{SO}_{4}{ }^{2}$ - ions, which is why Harned \& Owen [24] made this clear distinction between thermodynamic "components" and "constituents":

"In solutions of an electrolyte, electro-neutrality imposes the condition that the number of mols of the individual ionic species cannot be varied independently. We must be careful, therefore, to refer to ionic species as constituents of the solution rather than as components, so that the latter term may retain the precise meaning assigned to it by Gibbs. A component is an independently variable constituent of a solution. Thus, in the system $\mathrm{NaCl}$ and $\mathrm{H}_{2} \mathrm{O}$ there are two components whose chemical potential can be measured by the application of thermodynamics alone. They are, of course, $\mathrm{NaCl}$ and $\mathrm{H}_{2} \mathrm{O}$. Although the ionic constituents $\mathrm{Na}^{+}$and $\mathrm{Cl}^{-}$are of fundamental importance in determining the behavior and properties of the system, their concentrations are not independent variables. Thermodynamics does not permit the evaluation of the chemical potentials, free energies, activities, etc., of the individual ionic species. In spite of this limitation it is advantageous to express a number of thermodynamic developments in terms of "hypothetical" ionic activities, with the strict understanding that only certain ionic activity products, or ratios, have any real physical significance."

Ions exist, but they cannot be manipulated independently in macroscopic quantities, as neutral electrolytes can, without accompanying, charge-compensating counterions. Thermodynamics simply reflects this reality. And even the Bates-Guggenheim paper [8], which formed the basis of the IUPAC $\mathrm{pH}$ recommendation [6], acknowledges its approximate status in

"The rest of this report is concerned with the interpretation of $\mathrm{pH}$ values as approximations to thermodynamic quantities."

That does not imply that we cannot (or even should not) build theoretical models of interionic interactions, just that they can only be tested experimentally by comparison with directly measurable, thermodynamic quantities of electroneutral entities, as in combinations such as $a_{ \pm} \equiv \sqrt{a_{+} a_{-}}$. However, as described in section 1.1 of [3], Lewis \& Randall [25] could not find a way to determine the separate, single-ion activities $a_{+}$and $a_{-}$, or the corresponding activity coefficients $\gamma_{+}$and $\gamma_{\text {. }}$. And that was also the reason why Sørensen \& Linderstrøm-Lang [26] concluded that

" it would seem premature at present to introduce the activity principle for measurements of hydrogen ions in biochemical investigations generally"

although one will not find this opinion reflected in the IUPAC recommendation [6], which instead referred to this Sørensen \& Linderstrøm-Lang paper [26] as their sole justification for concluding that

“... it has been accepted that it is more satisfactory to define $\mathrm{pH}$ in terms of the relative activity of hydrogen ions in solution"

Some justification! One would assume (or rather: expect) that a controversial yet consequential IUPAC recommendation would rest on a firmer scientific basis. But if so, IUPAC certainly did not make that case. 


\section{Illustrating electrochemical obscurity}

\subsection{A simple argument}

We now consider some of the examples of electrochemical obscurities kindly collected by Prof. Kakiuchi [2] regarding the correct use of the Nernst equation. A careful definition of symbols and concepts can often avoid obscurities and possibly resulting misconceptions. I will use the equation numbers used by Prof. Kakiuchi, such as (2), which are readily distinguished from my double-digit numbering scheme, as in eq. (2.4), or the triple-digit numbering of Hamer $\& \mathrm{Wu}$ [27], and I will refer to his cell schematics with boldfaced Roman numbers, such as I. Let me start with his example in section 3 of [2], which opens with the statement

"In electroanalytical chemistry, single ion activities appear in the Nernst equation, ${ }^{6}$

$$
E=E^{\mathrm{o}}+(R T / F) \ln \left(a_{\mathrm{Ox}} / a_{\mathrm{Rd}}\right)
$$

where $E$ is the cell voltage of the following cell (the potential of the right-hand-side electrode with respect to the left), and $a_{\mathrm{Ox}}$ and $a_{\mathrm{Rd}}$ are the activities of $\mathrm{Ox}$ and Rd in the solution.

$$
\text { Reference electrode | Sample solution | Working electrode I }
$$

$E^{0}$ is the standard potential for the reaction in the test solution phase between the redox couple Ox and Rd,

$$
\mathrm{Ox}+\mathrm{e}^{-}=\mathrm{Rd}
$$

At least one of Ox and Rd is ionic species, and hence its activity is a single ion activity."

Point made, case closed! Or maybe not yet quite, because there are some inherent problems with the above-quoted statements. The electron $\mathrm{e}^{-}$resides in the metal rather than in the solution phase. And the cell voltage $E$ in cell $\mathbf{I}$ was explicitly defined as "the potential of the right-hand-side electrode with respect to the left", i.e., as a potential difference between the working and reference electrodes, and therefore should reflect the electrochemical properties of both electrodes contacting the sample solution. As written here, however, eq. (2) is like the sound of one hand clapping. And since the Nernst equation supposedly describes an electro-chemical equilibrium, how can $E^{0}$ be defined by an electrode reaction? That distinction is crucial here, because (electro)chemical rate expressions (such as eq. (3) according to its accompanying text) need not be electroneutral in its chemical components, whereas (electro)chemical equilibrium expressions must be. Even the sign = used is unnecessarily ambiguous: reaction rates are best denoted by single, directional arrows, such as $\rightarrow$, and equilibria (typically written in terms of balancing over-all reaction rates in opposite directions) by double arrows of equal length, as in $\leftrightarrows$.

Equilibrium potential differences $E$, including their standard values $E^{0}$, are always measured in the virtual absence of an electrical current, in order to ensure the equality of interfacial and bulk concentrations and/or activities, i.e., to avoid so-called "concentration polarization". For that very reason, typical $\mathrm{pH}$ meters (or other, general-purpose electrometers) are designed to have very high input resistances, so that they can measure equilibrium potentials with sufficient accuracy. 
That is also why $\mathrm{pH}$ meters and electrometers have no difficulty measuring potential differences across cells incorporating a glass electrode, which typically has an internal resistance of the order of $100 \mathrm{M} \Omega$. The electrons $\mathrm{e}^{-}$in eq. (3) must come from a metal electrode, whereas Ox and Rd are "in the solution", so that any net "reaction" described by eq. (3) must represent a flow of current through the cell and its external measuring circuit. The high input resistance of $\mathrm{pH}$ meters and general electrometers is usually of the order of at least $10^{11} \Omega$, as needed to measure with sufficient accuracy (here for the sake of the argument assumed to be to $\pm 0.1 \%$ ) the potential difference $E$ across a cell with such a glass membrane.

For a driving force $E$ of the order of $1 \mathrm{~V}$ or less, and a circuit resistance of the order of $10^{11}$ $\Omega$ or more, metal electrons cannot participate to any meaningful extent as reagents in a "reaction ... between the redox couple Ox and Rd", whatever that means, because the current involved would be at most of the order of $10^{-11} \mathrm{~A}$, or $10^{-11}$ Coulombs per second. Because $1 \mathrm{~F} \approx 10^{5} \mathrm{C} \mathrm{mol}^{-1}$, that will yield $10^{-16}$ moles per second, or approximately 3 nanomoles per year, or all of 0.3 micromoles per century! Some chemical reaction rate!

The argument [2] that "At least one of $\mathrm{Ox}$ and $\mathrm{Rd}$ is an ionic species, and hence its activity is a single ion activity", is clearly wrong, because based on an incorrect form of the Nernst equation.

\subsection{A corrected argument}

Didactically, it would seem counterproductive to start off in section 3 of [2] with the above, rather defective example but, in all fairness, eq. (2) was subsequently amended in section 4 of [2], which unapologetically begins as follows:

“ In fact, the Nernst equation, represented as (2) or (5), is not thermodynamically rigorous. Equation (6) is valid only when the liquid junction potential between the solution (1) and the reference electrode is the same as that of (2) and the reference electrode. As there is no way to accurately know the magnitude of the liquid junction potential, which is nonthermodynamic, eq. (6) premises the nonthermodynamic assumption.

In the first place, an electrochemical cell is not a thermodynamic device [13]. The cell is realized by splitting a redox reaction between two redox couples, $\mathrm{Ox}_{1} / \mathrm{Rd}_{1}$ and $\mathrm{Ox}_{2} / \mathrm{Rd}_{2}$,

$$
\mathrm{Ox}_{1}+\mathrm{Rd}_{2}=\mathrm{Rd}_{1}+\mathrm{Ox}_{2}
$$

into two half-cell reactions, each of which proceeds at one of the two electrodes,

$$
\mathrm{Ox}_{1}+\mathrm{e}^{-}=\mathrm{Rd}_{1}
$$

and

$$
\mathrm{Ox}_{2}+\mathrm{e}^{-}=\mathrm{Rd}_{2}
$$

Unfortunately, this makes matters only more confusing. Is the Nernst equation not thermodynamically rigorous, or does this statement apply only to its representations in [2] as eqs. (2) and/or (5)? If eq. (5) is not rigorous, how about eq. (6), which uses eq. (5) twice? Where does "...the liquid junction potential between the solution (1) and the reference electrode..." come from, or "that of (2) and the reference electrode"? The cell described in scheme I shows no such junction, nor is one needed. This question is especially relevant when that "liquid junction potential ... is nonthermodynamic". 
Apparently the last question is answered in the next paragraph in [2] by the categorical statement that "an electrochemical cell is not a thermodynamic device", followed by a reference to a paper published only in Japanese. Maybe so in voltammetry, but in potentiometry?

Why is an "Inner solution" suddenly interposed between the Reference electrode and the Sample solution in cell II? Is there a similar one between the Sample solution and the Working electrode? Should the (apparently different) characteristics of the "Reference" and "Working" electrodes not be explained first?

And why is it necessary to introduce a "liquid junction" between two or even three solution compartments? Heyrovský's Nobel-prize-winning polarographic measurements did not feature liquid junctions, and neither did the equilibrium adsorption studies by Gouy. What, then, makes the isolation of two redox couples

"an indispensable prerequisite for any electrochemical cell"

and in what respect does this "more precisely" represent cell I, which only shows one redox couple in its eq. (3), and no liquid junction?

The text between eqs. (7) and (8) in [2] reiterates the problem identified earlier in connection with eq. (3), viz. the implication that the Nernst equation requires a net electron transfer reaction to proceed at each of the two electrodes. This is reinforced in the next paragraph:

"Without the splitting, the reaction (7) proceeds spontaneously within the cell and the energy cannot be made use of externally as electrical energy. The isolation of the two redox couples, which is an indispensable prerequisite for any electrochemical cell, ${ }^{7}$ necessarily brings about the junction of the two electrolyte solutions. At this junction, the liquid junction potential inevitably emerges on one level or another. The cell I is more precisely,

Reference electrode | Inner solution | Sample solution | Working electrode

II

The liquid junction resides at the interface between the inner solution and the sample solution."

From where came the need to generate externally usable electrical energy? Is this a general requirement? Does it apply to potentiometric equilibrium measurements such as those of $\mathrm{pH}$ ? What made cell II "more precisely" represent cell I, even when cell II has many attributes not present in cell I? Moreover,

“Cell II more precisely is represented as

Reference electrode | Inner solution | Salt bridge | Sample solution | Working electrode

III "

so that cell I, after first having been morphed into cell II, is now more precisely represented by cell III. The cell, as well as the explanation of its potential, gets more and more convoluted!

And what will guide the reader to the conclusion that

“... the liquid junction potential inevitably emerges on one level or another."

when footnote 7 mentions several well-known electrochemical cells, such as the lead-acid battery, the Weston element, and the Harned cell, which neither have nor need any liquid junction. 
Moreover, what justifies the characterization in [2] section 5.1 that

"... the use of an electrochemical cell that apparently does not have a liquid junction ... is a halfway measure."

when the latest IUPAC $\mathrm{pH}$ recommendation [6] states that

"primary $\mathrm{pH}$ standard values can be determined from electrochemical data from cells without transference using the hydrogen gas electrode, known as the Harned cell. These primary cells have good reproducibility and low uncertainty. Cells involving glass electrodes and liquid junctions have considerably higher uncertainties, ..."

and, more specifically, that

"The only method that meets the stringent criteria of a primary method of measurement for measuring $\mathrm{pH}$ is based on the Harned cell..."

\subsection{Deriving the correct Nernst equation for cell I}

Fortunately, we need not go into the many unexplained, utterly confusing details of the examples considered so far, because section 5.2 of ref. [2] can be used to derive the correct form of eq. (3), and to identify its error as caused by careless and/or misleading notation and nomenclature. It considers cell III, and more specifically deals with

$$
\mathrm{Ag}|\mathrm{AgCl}| \text { aqueous } 0.01 \mathrm{M} \mathrm{HCl} \mid \text { salt bridge solution | aqueous } x \mathrm{M} \mathrm{HCl}, \mathrm{H}_{2} \mid \mathrm{Pt}
$$

IV

in which both the working (platinum) and reference $(\mathrm{Ag} / \mathrm{AgCl})$ electrode are finally chemically specified. They are immersed in an aqueous $\mathrm{HCl}$ solution, the $\mathrm{Pt}$ "working" electrode is saturated with $\mathrm{H}_{2}$ at standard temperature and pressure, and the silver "reference" electrode is coated with $\mathrm{AgCl}$, in contact with an aqueous solution of $0.01 \mathrm{~mol} \mathrm{HCl}$ per $\mathrm{L}$ of solution. For the sake of internal consistency we will again use molal (rather than molar) concentrations. Furthermore we will assume that the so modified formalism of [2] section 5.2 holds for any non-negative value of $x$, and for any (so far unspecified) composition of the salt bridge. We will therefore simplify matters by instead applying scheme $\mathbf{I V}$ to the cell

$$
\mathrm{Ag}|\mathrm{AgCl}| x \mathrm{~mol} \mathrm{~kg}^{-1} \mathrm{HCl}\left|x \mathrm{~mol} \mathrm{~kg}^{-1} \mathrm{HCl}\right| x \mathrm{~mol} \mathrm{~kg}^{-1} \mathrm{HCl}, \mathrm{H}_{2} \mid \mathrm{Pt}
$$

where the aqueous solutions in the "inner", "salt bridge", and "sample" compartments all have the same "swamping" $\mathrm{HCl}$ concentration of $x$ moles of $\mathrm{HCl}$ per $\mathrm{kg} \mathrm{H}_{2} \mathrm{O}$, and differ only in the concentrations of their minor constituents, because some silver-chloro-complexes will be present at low concentrations in the "inner" solution, and some dissolved $\mathrm{H}_{2}$ in the "outer" solution. Note that $\mathrm{H}_{2}$ would not be needed if we were to use a glass electrode instead, and anyway, as an uncharged species, $\mathrm{H}_{2}$ can be expected to have only a quite small, rather indirect influence on interionic interactions, perhaps by affecting the dielectric permittivity of the solution [28]. The same applies to the slightly soluble $\mathrm{AgCl}$. The minor concentrations of $\mathrm{AgCl}_{2}{ }^{-}, \mathrm{AgCl}_{3}{ }^{2-}$, etc. can be estimated from their known equilibrium constants, and can be kept away effectively from the $\mathrm{H}_{2} \mid$ Pt electrode by using a stopcock in capillary tubing to make solution contact only during the measurements, and by avoiding any hydrostatic pressure differences which might cause net solution flow through that capillary. 
We again quote ref. [2], this time from its section 5.2, as:

“... The cell voltage, that is, the potential of the hydrogen electrode on the right-hand side with respect to the silver|silver chloride electrode on the left-hand side, $E$, is expressed in the following Nernst equation,

$$
E=E_{\left.\mathrm{H}^{+}\right|_{\mathrm{H}_{2}} ^{\mathrm{o} r e f}}+\frac{R T}{F} \ln a_{\mathrm{H}^{+}}
$$

where $E_{\mathrm{H}^{+} \mid \mathrm{H}_{2}}^{\mathrm{o} \text {,ref }}$ is the standard potential of the working electrode. The subscript " $\mathrm{H}^{+} \mid \mathrm{H}_{2}$ " designates that it is the standard potential of the hydrogen electrode, and the superscript "ref" indicates that this standard potential depends on the reference electrode."

and a little further-on (where ILSB stands for Ionic Liquid Salt Bridge):

“ $E_{\mathrm{H}^{+} \mathrm{H}_{2}}^{\mathrm{o}, \text { ref }}$ consists of the difference $\Delta \phi_{\mathrm{W} 1 \mid \mathrm{ILSB}} \Delta \phi_{\mathrm{W} 2 \mid \mathrm{ILSB}}$ in the liquid junction potentials on both side of the salt bridge and the electrode potential of the silver| silver chloride electrode, i.e.,

$$
E_{\mathrm{H}^{+} \mid \mathrm{H}_{2}}^{\mathrm{o}, r e f}=E_{\mathrm{Ag} \mid \mathrm{AgCl}}^{\mathrm{o}}+\frac{R T}{F} \ln a_{\mathrm{Cl}^{-}}^{\mathrm{III}}+\Delta_{\mathrm{ILSB}}^{\mathrm{W}(\mathrm{III})} \phi-\Delta_{\mathrm{ILSB}}^{\mathrm{W}(\mathrm{V})} \phi
$$

Combining eqs. (10) and (12) would indeed yield the typical Nernstian expression for the potential difference across cell $\mathbf{I V}^{\prime}$, assuming that the terms $\Delta \phi$ properly describe the resulting (but unknown, immeasurable, and inherently time-dependent) potential differences $\Delta \phi$ across its liquid junctions. With that proviso, eq. (12) can be combined with eq. (10) to yield

$$
E=-E_{\mathrm{Ag} \mid \mathrm{AgCl}}^{\mathrm{o}}+\frac{R T}{F} \ln a_{\mathrm{H}^{+}}+\frac{R T}{F} \ln a_{\mathrm{Cl}}+\Delta_{\mathrm{ILSB}}^{\mathrm{WI}} \phi-\Delta_{\mathrm{ILSB}}^{\mathrm{W} 2} \phi
$$

We now apply eq. (12') to the junction-free cell I introduced in section 3 of [2]. And because this cell shows no liquid junctions, we may set $\Delta_{\text {ILSB }}^{\mathrm{W} 1} \phi-\Delta_{\text {ILSB }}^{\mathrm{W} 2} \phi$ in eq. (12') equal to zero, and thereby obtain for the potential difference across the junction-free cell $\mathbf{I}$ :

$$
E=-E_{\mathrm{Ag} \mid \mathrm{AgCl}}^{\mathrm{o}}+\frac{R T}{F} \ln a_{ \pm, \mathrm{HCl}}^{2}
$$

Comparison of eq. (12") with

$$
F\left(E-E^{\mathrm{o}}\right) / R T=-\ln a_{\mathrm{Ag}^{+}}
$$

then shows that eq. (5) is either wrong or, at least, grossly misleading, because its "standard potential" $E^{\mathrm{o}}$ as well as its logarithmic term conceal its crucial dependence on the chloride activity. In other words, the reader of [2] is not warned that the symbols $E^{\circ}$ and $E_{\mathrm{Ag} / \mathrm{AgCl}}^{\mathrm{o}}$ are used here with quite different meanings, and that eq. (2) is inapplicable to a junction-free cell because the $\mathrm{AgCl}$-coated silver wire is in direct contact with the chloride ions! Consequently, the argument that the Nernst equation, as applied to cell II, "must contain at least one single ion activity, and hence its activity is a single ion activity" is not valid: it contains the square root of the product of two single ion activities, of opposite sign, i.e., a mean salt activity $a_{ \pm}$, as reflected in the equivalence of eqs. $\left(12^{\prime}\right)$ and $\left(12^{\prime \prime}\right)$ after the term $\Delta_{\mathrm{ILSB}}^{\mathrm{W} 1} \phi-\Delta_{\mathrm{ILSB}}^{\mathrm{W} 2} \phi$ has been dropped. So much for the single ion activity and the Nernst equation. We will return to this question in a separate communication. 
Of course you need not take my word for this. The primary potentiometric measurements on aqueous $x$ mol kg${ }^{-1} \mathrm{HCl}$, which were all made in junction-free cells with $\mathrm{Pt} \mid \mathrm{H}_{2}$ and $\mathrm{Ag} \mid \mathrm{AgCl}$ electrodes, are those by Harned \& Ehlers [29] and by Bates \& Bower [30], and both papers use the Nernst equation in the form

$$
E=E_{\mathrm{Ag} \mid \mathrm{AgCl}}^{\mathrm{o}}-\frac{R T}{F} \ln a_{\mathrm{H}^{+}} a_{\mathrm{Cl}^{-}}=E_{\mathrm{Ag} \mid \mathrm{AgCl}}^{\mathrm{o}}-\frac{R T}{F} \ln \left(m_{\mathrm{H}^{+}} \gamma_{\mathrm{H}^{+}} m_{\mathrm{Cl}^{-}} \gamma_{\mathrm{Cl}^{-}}\right)
$$

which is identical to eq. (12"), and clearly shows that the cell potential difference $E$ in Fig. 1 of [2] should respond to the activities of both $\mathrm{H}^{+}$and $\mathrm{Cl}^{-}$, and could then be used to determine $\gamma_{ \pm}$ (since $m_{ \pm}=x$ was known) and, by extrapolation, $E^{0}$ for this cell, but cannot be used to determine $a_{\mathrm{H}^{+}}, a_{\mathrm{Cl}^{-}}, \gamma_{\mathrm{H}^{+}}$, and/or $\gamma_{\mathrm{Cl}^{-}}$individually.

\section{Odds and end}

\subsection{Consider Fig. 1}

What was behind the rhetorical question

"However, is it possible to understand satisfactorily the properties of electrolyte solutions only in terms of the properties of salts, as claimed by de Levie?"

Again, there seems to be some confusion here. Thermodynamics provides a framework of strictly mathematical relations between measurable parameters, but understanding the meaning of the resulting numerical values must come from models or other extra-thermodynamic insights.

Even more puzzling is the paragraph following Fig. 1, which reads:

"From this figure, it can be seen that the value of $\gamma_{ \pm \mathrm{HX}}$ depends on the type of $\mathrm{X}^{-}$. For example, the values of $\gamma_{ \pm \mathrm{HX}}$ for $\mathrm{HCl}, \mathrm{HBr}$, and $\mathrm{HI}$ at $0.5 \mathrm{~mol} \mathrm{~kg}^{-1}$ are $0.759,0.790$, and 0.845 respectively [3]. It seems impossible to explain this difference in these values, and also the different dependences on the ionic strength in Fig. 1 in terms of only the properties of salts."

I can best refer the reader to the standard works on this topic, such as by Harned \& Owen [25] and by Robinson \& Stokes [31]. The data shown in Fig. 1 of [2] were taken from a critical selection, made and recalculated by Hamer \& Wu [27], of experimental results at $25^{\circ} \mathrm{C}$, and only differ from those in other compilations (if at all) in their least-significant $\operatorname{digit}(\mathrm{s})$. In introducing their approach, Hamer \& Wu specifically list the Nernst equation for the sample cell

as

$$
\mathrm{Pt}, \mathrm{H}_{2}(g)|\mathrm{HCl}(m)| \mathrm{AgCl}(s), \mathrm{Ag}(s)
$$

$$
E=E^{\mathrm{o}}-\frac{R T}{F} \ln \left(m_{\mathrm{H}^{+}} \gamma_{\mathrm{H}^{+}} m_{\mathrm{Cl}^{-}} \gamma_{\mathrm{Cl}^{-}}\right)
$$

None of the above-quoted papers [29,30], books [24,31] or data compilations [27] show expressions for the potential of such a cell as either eq. (2) or (5) of [2]. And since all those other sources show an explicit dependence of $\gamma_{ \pm \mathrm{HX}}$ on $\gamma_{\mathrm{X}^{-}}$, little remains of the statement made in the paragraph following Fig. 1 of [2] that 


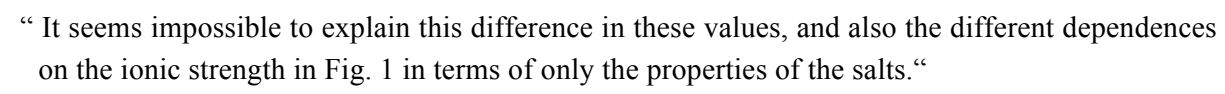

The electrolytes shown in Fig. 1 of [2] have a common cation, and only differ in their anions. Is the implication of the above quote that these data for different strong acids should all show the same dependency on ionic strength? In other words, that all electrolytes have equal mean activity coefficients at equal ionic strengths of up to about 5 molal? Certainly, Lewis and Randall [32] never claimed such equality beyond 0.1 molal, and the data in Fig. 1 obviously don't show that either! So what is at question here: the validity of the experimental data, their interpretation, or what?

\subsection{Some hedging!}

The obscurities described in section 3 may have different origins. I could not agree more with Prof. Kakiuchi's statement regarding

"the obscure interpretation of the Nernst equation ... which has long been applied to a working electrode"

rather than

“... to the potential difference between a 'working' and 'reference' electrode".

When the platinum electrode responds to the $\mathrm{H}^{+} / \mathrm{H}_{2}$ equilibrium, and the $\mathrm{Ag} / \mathrm{AgCl}$ electrode to that between $\mathrm{Ag}$ and $\mathrm{Ag}^{+}$in $\mathrm{AgCl}$, it is tempting to ascribe different electrode potentials to these two electrodes. However, Gibbs already noticed in his earlier-quoted letter to Bancroft [17] that

“... the consideration of the electrical potential in the electrolyte, and especially the consideration of the differences of potential in electrolyte and electrode, involve the consideration of quantities of which we have no apparent means of physical measurement, while the difference in potential in "pieces of metal of the same kind attached to the electrodes" is exactly one of the things which we can and do measure."

But then, rather uncharacteristically, Gibbs continued:

"Nevertheless, with some hedging in regard to the definition of the electrical potential, we may apply

$$
V^{\prime \prime}-V^{\prime}=\alpha_{a}\left(\mu_{a}^{\prime}-\mu_{a a^{\prime \prime}}\right)
$$

to points in electrolyte (') and electrode ("). This gives

say,

$$
V^{\prime \prime}-V^{\prime}=\alpha_{a}\left(B_{a}^{\prime}+\frac{A t}{M_{a}} \log \gamma_{a}-\mu_{a a^{\prime \prime}}\right)
$$

$$
V^{\prime \prime}-V^{\prime}=\frac{\alpha_{a} A t}{M_{a}} \log \frac{\gamma_{a}}{G}
$$


So here is the slippery slope, shown us by none other than by Gibbs himself! Some of these problems indeed go back quite far!

\subsection{Some elementary statistics}

When a small difference is calculated as the sum and/or difference of two or more very much larger experimental quantities, such as $(222.49 \pm 0.2+120.9 \pm ? ?-342.9 \pm ? ?) \mathrm{mV}$ in [2] Fig. 3 , the resulting uncertainty estimate must of course include the "cancellation" errors in adding and subtracting such large, near-equal quantities. Where are the estimated errors in at least two those three quantities? And where is the statistical error in their difference $\Delta$ ? Why are such standard deviations missing in Fig. 3 of [2] and apparently ignored in computing the resulting error estimate of merely $-0.5 \mathrm{mV}$ in the liquid junction potential? And where is the error estimate of the term $-(R T / F) \ln \left(c_{\mathrm{HCl}} \times \gamma^{\left({ }^{(}\right)}{ }_{\mathrm{Cl}}\right)$ ? This appears to be the evidence supporting the claim made in the abstract of [2] that

"the single ion activity can be estimated with a reasonable certainty"

which led to the estimate in the Conclusions of [2] as

"... shown to be lowered to the level of ca. $0.5 \mathrm{mV}$."

Even so, not much appears to have changed in the 90 years since Güntelberg [34], admittedly a stickler for precision, wrote (in my translation from German into English):

\footnotetext{
"Many researchers have measured potentials in electrolyte solutions. In the great majority of cases, these studies have only determined the changes in potential due to changing one type of ions through the addition of a neutral electrolyte. Such studies can possibly serve as a first orientation, but are usually of little value for exact studies of the relations governing the relationships in mixtures, because our understanding of their necessarily occurring liquid junction potentials, as well as their reproducibility, is unfortunately still quite poor. Still one mentions the "elimination" of liquid junction potentials, and even of their "complete annihilation", but we can only diminish them, and their variability is usually assigned an uncertainty of $1 / 2$ to 1 millivolt $^{1}$ ). However, the individual differences between electrolytes of the same type observed in $0.1 \mathrm{M}$ solutions are usually measured as of the same order of magnitude. It is therefore absolutely necessary to avoid liquid junction potentials."

“1) Many researchers are therefore not worried about the liquid junction potential between $0.1 \mathrm{~N} \mathrm{KCl}$ and a saturated solution thereof, which is of the order of $0.8 \mathrm{mV}$."
}

Needless to say, Güntelberg [34] only used cells without liquid junctions.

\subsection{When to use single-ionic activity coefficients}

It is certainly not my intention to scare anyone away from using immeasurable quantities, such as complex numbers, electronic wave functions, or single-ionic activity coefficients, so let me specify when, in my opinion, they can be used, and when they shouldn't be.

Single-ionic activity coefficients are often necessary in modeling the interactions of ions with other solutes, be they ionic or molecular. A prime example of their proper experimental use was given in the Debye-Hückel model [35], where the model (here illustrated for the aqueous solution of a single strong 1,1-electrolyte) computed $\gamma_{+}$as reflecting the net effect of all 
coulombic interactions on a "central" cation, then applied the same model prediction to a central anion to find $\gamma_{-}$, and finally compared the resulting prediction for $\sqrt{\gamma_{+} \gamma_{-}}$with the measured mean activity coefficient, $\gamma_{ \pm}$.

The excellent fit so obtained between the model prediction and the thermodynamic data in the "limiting law" region of very low ionic strength [36], without using any adjustable parameters, facilitated its quick and near-general acceptance. The same test can of course be applied to other models, such as that proposed by Brønsted, which was further developed by Guggenheim and by Scatchard, or that of Pitzer. Brønsted emphasized the dominant interionic effect of counterions, which are readily and successfully modeled in terms of mean electrolyte activity coefficients and, incidentally, might be of interest here because they fully explain Fig. 1 in terms of counter-ion effects.

A prime example of the misuse of single-ionic modeling is in the assignment of a singleionic activity coefficient to a directly measurable equilibrium cell potential, as in IUPAC's $\mathrm{pH}$ recommendation, because macroscopic electroneutrality can neither be ignored nor wished away. The same applies to, e.g., eq. (5) in [2]. In treating equilibrium measurements, we cannot select which thermodynamic relationships are to our liking and which are not. They come as fully integrated package deals, not as individually selectable menu items.

\subsection{The effect of mixtures}

In solutions of a single electrolyte, the mean electrolyte activity coefficient must tend to 1 when its concentration tends to zero, and this requirement is generally assumed to hold also for single-ionic activity coefficients. The same applies to the Bates-Guggenheim model expression for $\gamma_{\mathrm{Cl}^{-}}$[8] which forms the basis of the IUPAC pH recommendation [6], which is modeled after the Debye-Hückel approach with the assumption that, for $I \leq 0.1 \mathrm{~mol} \mathrm{~kg}^{-1}, \gamma_{\mathrm{Cl}^{-}}=\gamma_{\mathrm{Na}^{+}}=\sqrt{\gamma_{ \pm, \mathrm{NaCl}}}$. However, as already explained in [3], and in much more detail by Harned \& Robinson [33], in electrolyte mixtures the Bates-Guggenheim relation does not apply to minor electrolytes in a "swamped" solution, even for $I \leq 0.1 \mathrm{~mol} \mathrm{~kg}^{-1}$, because the corresponding mean trace activity coefficients do not tend to 1 when the concentration of the corresponding trace constituent goes to zero while the concentration of the swamping electrolyte, and hence the ionic strength, remains constant. Moreover, a careful reading of Hückel's paper [28] will show that it does not support a single term $+b I$ for electrolyte mixtures. Given the rather narrow $\mathrm{pH}$ range of many biological samples, typically between 4 to 10 , and the correspondingly low hydrogen ion concentrations, this oversight is often consequential, and would be even more so at ionic strengths exceeding 0.1 mol kg ${ }^{1}$. This matter was discussed explicitly in section 3.5 and 3.6 of ref. [3].

\subsection{Concluding remarks}

Prof. Kakiuchi generously mentioned that, in my Prague lecture, I

"...explained a way of converting the activity to the concentration." but that,

"From a practical point, to the best of my knowledge, there is no general method of calculating the concentration from the activity." 
I will gladly subscribe to this sentiment when Prof. Kakiuchi means "from the single-ionic activity." However, when the mean activity $a_{ \pm}=\gamma_{ \pm} m_{ \pm}$of a single strong 1,1-electrolyte solute can be obtained by valid thermodynamic methods for a given mean molality $m_{ \pm}$, as in potentiometric measurements on cells without liquid junctions, one can usually calculate $m_{ \pm}$from $a_{ \pm}$provided that all relevant equilibrium constants of the system are known. Moreover, the results obtained during more than a century of measurements have shown that swamping with one or more concentrated, strong 1,1-electrolytes such as $\mathrm{KCl}, \mathrm{LiNO}_{3}$, or $\mathrm{LiClO}_{4}$, can indeed render $\gamma_{ \pm}$ essentially constant for minor components in aqueous electrolyte mixtures. The magnitude of any remaining dependence of $\gamma_{ \pm}$on $m_{ \pm}$can then be calculated, so that $\gamma_{ \pm}$can be computed as a function of $m_{ \pm}$using a thermodynamically valid protocol. In a separate publication we will illustrate how this approach can be used to determine $-\log \left(m_{\mathrm{H}^{+}}\right)$, as well as for the exact acid-base titration of arbitrary mixtures of monoprotic or polyprotic acids or bases in both sample and titrant.

Inorganic chemists [37,38] have long known and practiced swamping so as to minimize activity effects. This has allowed them to separate weak complexation from activity effects. And so have electroanalytical chemists, who perhaps didn't realize that their "supporting" electrolyte not only reduces ohmic voltage drops when their electrolyte solutions carry an electric current, but even in the absence of a significant cell current will stabilizes the "trace" activity coefficients of their minor electroactive solutes.

The measurements of polarographic wave heights, and of cyclic voltammetric peak currents, as directly proportional to the bulk concentrations of the electroactive species, likewise rely on a swamping inert electrolyte to make diffusion-controlled currents independent of activity coefficients. After all (but now using irreversible thermodynamics, because in this case we deal with non-equilibrium process), Fick's first law of diffusion specifies that the mass flux in a stationary solution is driven by a force proportional to the gradient of the electrochemical potential [22], and for ions therefore depends not only on the electric field, but also on the gradient in the natural logarithm of the activity. An inert, strong, swamping electrolyte reduces both gradients, so that the measured diffusional flux tends to become directly proportional to the solute concentration (rather than activity) of the electroactive species, and at the same time less dependent on the corresponding movements of other solute ions.

I therefore concur with the tenor of the final paragraph of [2], at least for voltammetry, but not necessarily for the reason given there. The current per se is not immune to activity effects, but in voltammetric measurements of mass-transport-limited currents, a large excess of "inert", swamping electrolyte is usually present, making the activity coefficients of the electroactive species essentially independent of their own bulk concentrations, which then yields a linear correlation between limiting current and bulk concentration.

In separate communications I will address some of the above points more explicitly. Suffice it to mention here that I neither possess nor search for a magic wand to convert an immeasurable single-ion activity into a verifiable single-ion concentration. To me, that would be a prototypical fool's errand. Here I am again in agreement with Prof. Kakiuchi, in that I don't foresee any such method, nor do I anticipate any need for it, because an undefined, immeasurable input value is not 
an attractive starting point for determining an experimental quantity. But deriving the hydrogen ion concentration from potentiostatic measurements of cells without liquid junctions is an entirely different, often quite doable matter.

\section{References}

[1] K. Kano, Rev. Polarogr. 61 (2015) 141-142.

[2] T. Kakiuchi, Rev. Polarogr. 61 (2015) 105-116.

[3] R. de Levie, Electrochim. Acta 135 (2014) 604-639.

[4] H. Friedenthal, Z. Elektrochem. 10 (1904) 113-119.

[5] S. P. L. Sørensen, Biochem. Z. 21 (1909) 131-304.

[6] R. P. Buck, S. Rondinini, A. K. Covington, E. G. K. Baucke, C. M. A. Brett, M. F. Camões, M. J. T. Milton, T. Mussini, R. Naumann, K. W. Pratt, P. Spitzer, G. S. Wilson, Pure Appl. Chem. 74 (2002) 2169-2200.

[7] M. F. Camões, Chem. Internat. 32 \#2 (2010) 6-7.

[8] R. G. Bates, E. A. Guggenheim, Pure Appl. Chem. 1 (1960) 163-168.

[9] A. K. Covington, R. G. Bates, R. A. Durst, Pure Appl. Chem. 57 (1985) 531-542.

[10] Lord Kelvin, Lecture on "Electrical Units of Measurement" (3 May 1883), published in Popular Lectures, Vol. I, p. 73.

[11] J. N. Brønsted, Det Kgl. Danske Videnskabernes Selsk. Skrifter (7) 12 (1914) 241-268.

[12] J. N. Brønsted, J. Am. Chem. Soc. 45 (1923) 2898-2910.

[13] R. G. Bates, in The Nature of Seawater, E. D. Goldberg, ed., Dahlem Workshop Reports (1975) 312-338.

[14] R. G. Bates, J. B. Macaskill, in Analytical Methods in Oceanography, T. R. P. Gibbs, ed., ACS Advances in Chemistry \# 147 (1975) 110-123.

[15] J. B. Macaskill, R. A. Robinson, R. G. Bates, J. Solution Chem. 6 (1977) 385-392.

[16] J. W. Gibbs, Trans. Conn. Acad. Sci. 3 (1875-1878) 108-248, 342-524. reprinted in The Collected Works of J. Willard Gibbs, Yale Univ. Press, New Haven 1948.

[17] W. D. Bancroft, J. Phys. Chem. 7 (1903) 416-427, reprinted in J. W. Gibbs, ref. 20, pp. 425-434.

[18] W. Nernst, Silliman Memorial Lectures at Yale University on "The Experimental and Theoretical Applications of Thermodynamics to Chemistry" (1907) p. 9.

[19] R. Clausius, Pogg. Ann. Phys. Chem. 125 (1865) 400.

[20] G. N. Lewis, Proc. Am. Acad. Arts Sci, 43 (1907) 259-273.

[21] M. Sastre, J. A. Santabella, J. Chem. Educ. 66 (1989) 403-404.

[22] E. A. Guggenheim, J. Phys. Chem. 33 (1929) 842-849.

[23] E. A. Guggenheim, J. Phys. Chem. 34 (1930) 1540-1543.

[24] H. S. Harned, B. B. Owen, The Physical Chemistry of Electrolyte Solutions, $3^{\text {rd }}$ ed., Reinhold, New York 1925, p. 7.

[25] G. N. Lewis, M. Randall, Thermodynamics and the Free Energy of Chemical Substances, MacGraw-Hill, New York (1923) pp. 379-383.

[26] S. P. L. Sørensen, K. Linderstrøm-Lang, Compt. Rend. Trav. Lab. Carlsberg 15 (1924) 1-40.

[27] W. J. Hamer, Y.-C. Wu, J. Phys. Chem. Ref. Data 1 (1972) 1047-1099.

[28] E. Hückel, Physik. Z. 26 (1925) 93-147.

[29] H. S. Harned, R. W. Ehlers, J. Am. Chem. Soc. 55 (1933) 2179-2193.

[30] R. G. Bates, V. E. Bower, J. Res. NBS 53 (1954) 283-290.

[31] R. A. Robinson, R. H. Stokes, Electrolyte Solutions, $2^{\text {nd }}$ ed., Butterworth, London 1959.

[32] G. N. Lewis, M. Randall, J. Am. Chem. Soc. 43 (1921) 1112-1154. 
[33] H. S. Harned, R. A. Robinson, Equilibrium Properties of Electrolyte Solutions 2: Multicomponent Electrolyte Solutions, Pergamon Press 1968.

[34] E. Güntelberg, Z. physik. Chem. 123 (1926) 199-247.

[35] P. Debye, E. Hückel, Physik. Z. 24 (1923) 185-206.

[36] J. N. Brønsted, V. K. La Mer, J. Am. Chem. Soc. 46 (1924) 555-573.

[37] G. Biedermann, L. G. Sillén, Arkiv för Kemi 5 (1953) 425-440.

[38] A. Braibanti, G. Ostacoli, P. Paoletti, L. D. Pettit, S. Sammartano, Pure Appl. Chem. 59 (1987) $1721-1728$. 